\title{
ARTICLE
}

\section{Monte Carlo calculations for the ATLAS cavern background}

\author{
Tatsumi Koi ${ }^{\mathrm{a}^{*}}$, David N. Brown ${ }^{\mathrm{b}}$, Alberto Fasso ${ }^{\mathrm{a}}$, Norman A. Graf ${ }^{\mathrm{a}}$, Paola Sala ${ }^{\mathrm{c}}$, Dennis H. Wright ${ }^{\mathrm{a}}$ and \\ Charles C. Young ${ }^{\mathrm{a}}$ \\ ${ }^{a}$ SLAC National Accelerator Laboratory, Menlo Park, CA, 94025; ${ }^{b}$ University of Louisville, KY, Louisville, 40292, USA; ${ }^{c}$ INFN \\ Milano, Milano, 20133, Italy
}

\begin{abstract}
A new application for simulating the ATLAS cavern background was developed. This was done using FLUGG, software that allows Geant4 geometry to be used within the FLUKA simulation framework. A Geant4 description of the ATLAS detector including its cavern was built from scratch for this application. In order to gain computing performance, our geometry is less detailed than that of GeoModel which is used in the full detector simulation, but good enough for the investigation of cavern background. Our geometry can also be used in a standalone Geant 4 simulation. Thus it is possible to perform unbiased comparisons between Geant4 and FLUKA using the same complex geometry. We compared neutron and photon fluxes using the FLUKA-FLUGG application with the result of Geant4 simulations based on the QGSP_BERT and QGSP_BERT_HP physics lists. In all cases the same set of initial collision 4-vectors produced by the PHOJET event generator was used. The result from the QGSP_BERT_HP physics list, which uses the High Precision (HP) neutron model, is similar to the result of FLUKA-FLUGG and the differences in the fluxes between them are within $40 \%$ in most regions of the ATLAS cavern. The result from the QGSP_BERT physics list, which does not include the HP model, does not agree with either of the previous two results.
\end{abstract}

\section{Keywords: bremsstrahlung; high-energy accelerator; Monte Carlo; dose distributions; event generator;} FLUGG; FLUKA; Geant4

\section{Introduction}

ATLAS[1] is a high energy physics experiment at the Large Hadron Collider (LHC) at the CERN laboratory in Geneva, Switzerland. Two counter-rotating proton beams, each accelerated to the highest currently available energy at the LHC, are made to collide at the center of the ATLAS detector. By analyzing the particles resulting from these collisions, the ATLAS collaboration seeks to discover fundamental particles and interactions of nature. The most interesting signals are mainly detected close to the primary proton-proton collision in terms of time. However there are also many low-energy, mostly neutral particles produced by the interaction of the collision products with the ATLAS detector and its shielding material. These particles are called cavern background, because they persist much longer than beam bunch spacing period and contaminate the signals of interest.

An extensive study of shielding in the ATLAS detector was carried out before its construction [2]; however there have been many modifications since then in many aspects. We have developed an application based on FLUGG which is an extension of FLUKA that

*Corresponding author. Email: tkoi@slac.stanford.edu allows a full Geant4 geometry, including magnetic field maps, to be used within the FLUKA framework. A Geant4 geometry of the as-built ATLAS detector was developed independently for this application. It is less detailed than the GeoModel description used in the full detector simulation, but sufficient for the study of cavern background.

The geometry may be used not only in the FLUGG application but also in pure standalone Geant4 simulations. This fact presents us with an exceptional opportunity to carry out unbiased comparisons between Geant 4 and FLUKA using the same complex geometry that reasonably represents a large, modern HEP detector. In addition to using identical geometries, we also use the same event files of the products of the proton-proton collisions produced by the PHOJET [3] event generator.

There of course exist comparisons among Monte Carlo codes [4], but most of them compare single interactions or processes and are usually done with simple geometries such as slabs or cylinders. In our comparison, we use a reasonably realistic geometry $24 \mathrm{x}$ $48 \mathrm{~m}$ in size and weighing 7000t. These numbers do not take into account the surrounding cavern and shielding materials which we included in the simulation geometry. With mesh scoring in both simulations, photon and neutron fluxes are compared throughout the 
entire ATLAS cavern with a granularity of $10 \mathrm{~cm} \times 10 \mathrm{~cm}$ in the R-Z dimensions.

We describe the FLUKA-FLUGG application in the next section, the setup of the simulations for the comparisons and its results in the following section, and the conclusions in the final section.

\section{Simulation for ATLAS cavern background}

\subsection{FLUGG, FLUKA and Geant4}

FLUKA[5] is a fully integrated particle physics Monte Carlo simulation package and Geant4[6] is a toolkit for the simulation of the passage of particles through matter. GEANT4 has his origin in HEP experiments, FLUKA has strong roots in shielding and radiation protection studies, however both have since been extended and applied to medical, shielding, space sciences and other fields. The two codes approach user aspects of simulations differently. As a fully integrated package, FLUKA expects users to run their simulations within the FLUKA framework. On the other hand, Geant4 takes a toolkit approach, designed for working within other frameworks as well as in standalone mode. FLUGG (FLUKA Geant4 Geometry) exploits this feature of Geant4. It is an extension of FLUKA which enables the use of Geant4 geometry within the FLUKA framework by encapsulating Geant4 navigation. All other ingredients of the FLUKA framework, namely physics models, tracking, scoring, possibly biasing,

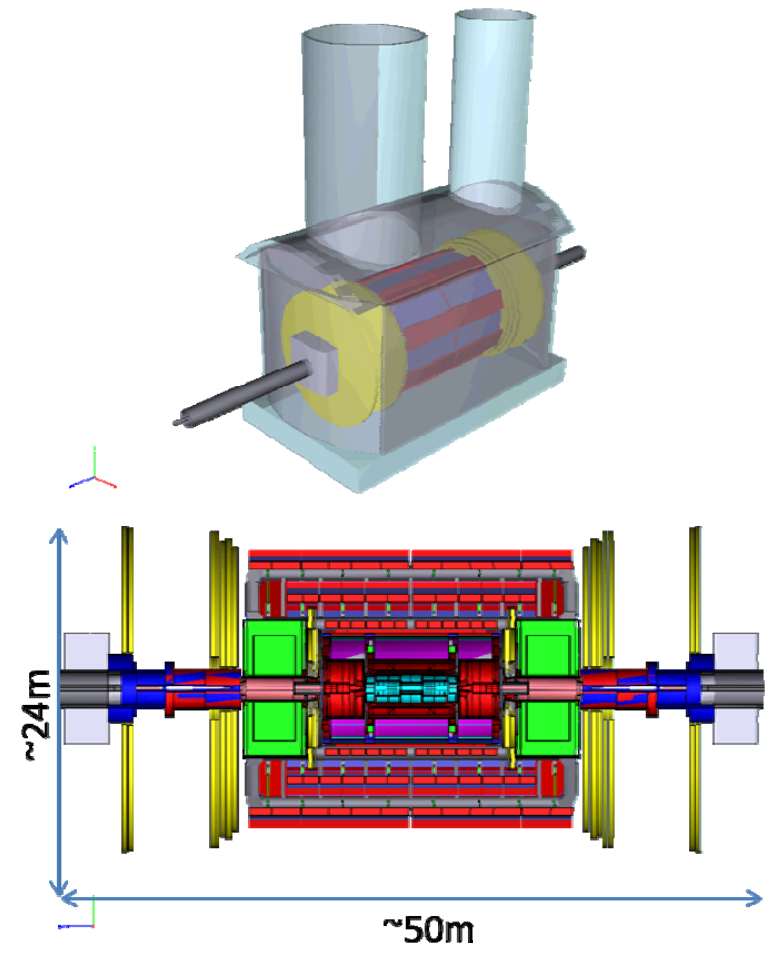

Figure 1. Visualizations of the geometry for Atlas cavern background; Upper panel shows cavern and its accesses shafts with beam line and detector. Detector side view is shown in lower panel. remain unchanged and fully accessible.

\subsection{New cavern background geometry}

The geometry developed for these comparisons was constructed mainly from boxes and cylinders in order to optimize computing performance. Where essential, more complex shapes such as cones, trapezoids, polygons and Boolean volumes were also used. The number of volumes in the geometry is about 5000, much smaller than the $\sim 1 \mathrm{M}$ in GeoModel. This benefits computing speeds and other resource consumption. Figure 1 shows the geometry we developed for the ATLAS cavern background study.

\section{Calculation setup and its results}

\subsection{FLUKA-FLUGG setup}

We used version 2011.2.14 (2012 Aug) of FLUKA. Important commands to describe our setup are "GLOBAL" and "DEFAULTS". The "fully analogue" option is selected in "GLOBAL" and "PRECISIO" is used for precision simulations in "DEFAULTS". With these commands, low energy neutron transportation is activated and analogue sampling is systematically carried out whenever it is possible. Neutrons below 20 $\mathrm{MeV}$ down to thermal energies are transported by a multigroup algorithm with the 260-group library that is distributed with the code.

Production thresholds are set to $10 \mathrm{keV}$ for gamma and $100 \mathrm{keV}$ for $\mathrm{e}^{-}$and $\mathrm{e}^{+}$.

It should be noted that this set-up is optimized for our cavern background study and produces un-weighted tracks. It is not ideal for general shielding calculations.

\subsection{Standalone Geant4 setup}

Version 9.5.p01 (March 2012) of Geant4 was used. We ran the simulation with QGSP_BERT and QGSP_BERT_HP physics lists, which define the physics processes of the simulation. QGSP_BERT consists in part of the Quark Gluon String (QGS) model with the Pre-compound interface as the high energy model, and the Bertini-like cascade as the low- and medium-energy model. This is the physics list used by most of the LHC experiments in their production Monte Carlos.

A relatively simple model for the low energy neutron transportation in QGSP_BERT physics list, that rooted in GEISHA hadronic package of Geant3, is replaced to the High Precision (HP) neutron model in the QGSP_BERT_HP physics list. The High Precision model is data-driven, using the G4NDL4.0 data files to provide continuous neutron transportation below $20 \mathrm{MeV}$ down to thermal energies. G4NDL4.0 is equivalent in data to ENDF/B-VII.0.

The Geant4 production threshold is defined by a range value, which is converted to a different energy threshold for each material. The physics list default value of 0.7 $\mathrm{mm}$ is used in these calculations. 


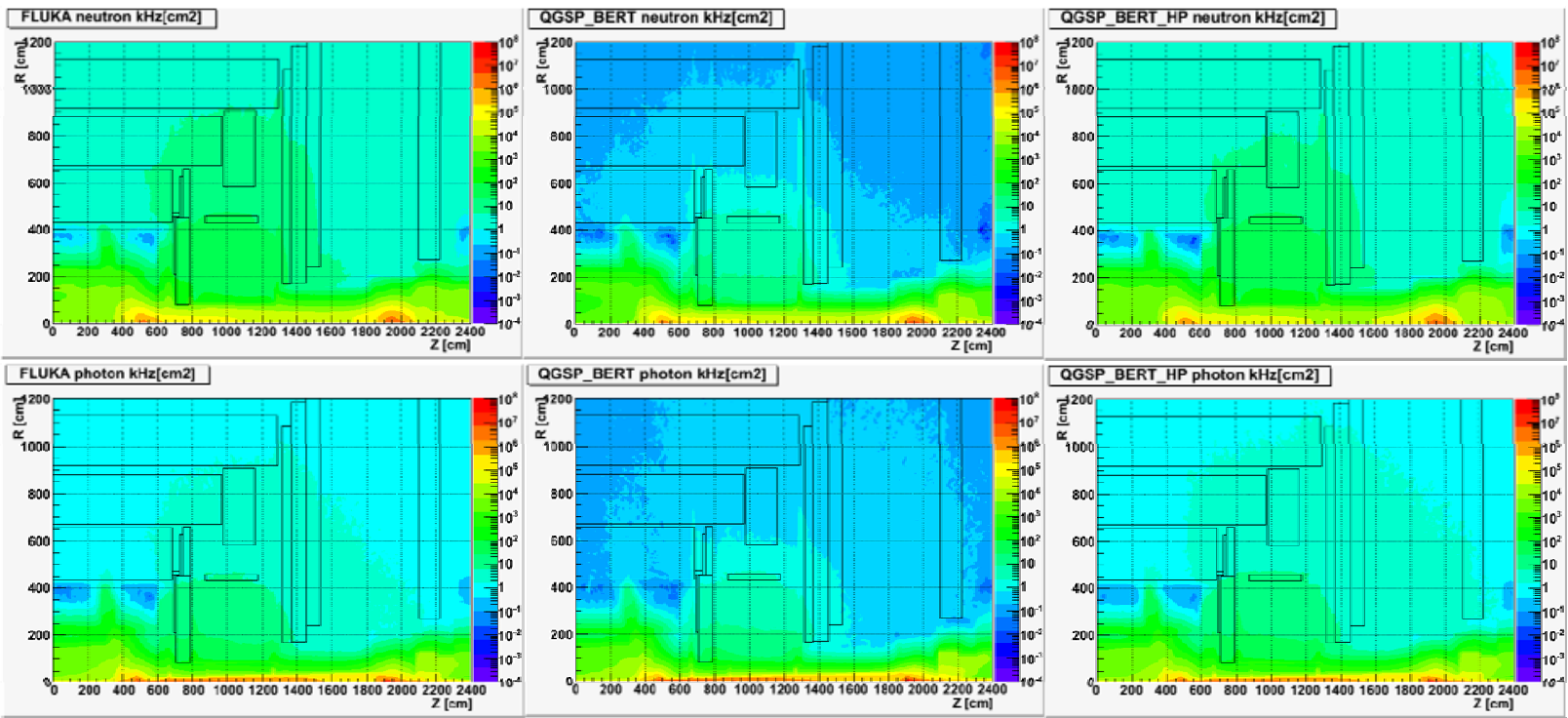

Figure 2. Neutron and photon flux map for each simulation. Boxes indicate the location of the muon systems. We use a same color scale in all plots and the upper and lower limit of the color scale is $10^{8}$ and $10^{-4}$ respectively.

\subsection{Scorer}

Geometry-independent cylindrical mesh scorers are used in all calculations. The scorers accumulate particle flux from 0 to $12 \mathrm{~m}$ in $\mathrm{R}$ and 0 to $24 \mathrm{~m}$ in $\mathrm{Z}$ with a mesh size of $10 \mathrm{~cm}$ in both $\mathrm{R}$ and $\mathrm{Z}$. The origin is the beam collision point and the $\mathrm{Z}$ axis is parallel to the beam line. The USRBIN card of FLUKA and the command line scorer of Geant 4 are used in configuring the scorers. The scorers record neutron and photon fluxes for all energies. These quantities are the most important for cavern background studies and it is relatively easy to accumulate high statistics to compare to other quantities. It was predicted from a small sample test that the number of tracks of other particle like muons, electrons, protons and other hadrons in each mesh are much lower than neutrons and photons. Due to the relative rarity of charged particles in background studies, a rather large statistical sample of events would be required. Since our study concentrated on neutral particles we did not feel that such a sample was warranted. The flux of photons $>1 \mathrm{MeV}$ is also recorded to check the potential impact of differences in the treatment of production cut values between FLUKA and Geant4.

\subsection{Input events}

Minimum bias p-p collision events calculated by the event generator PHOJET are used as input by the calculation. The total collision energy is $7(3.5+3.5) \mathrm{TeV}$. Exactly the same event data are used in all calculations.

\subsection{Results}

For each FLUKA-FLUGG, QGSP_BERT and QGSP_BERT_HP case, 1000 jobs were run, each job consisting of $10 \mathrm{p}-\mathrm{p}$ collision events. Thus the total number of events per case should be 10,000. Figures 2 through 4 show the results of the calculations. A total p-p cross section of $80 \mathrm{mb}$ and luminosity of $10^{34} \mathrm{~cm}^{-2}$ $\mathrm{sec}^{-1}$ were used in producing Figure 2. The cross section is not precisely correct, but has been used here for historical reasons. The luminosity is the design value of the LHC.

Figure 2 shows the neutron and photon flux maps of each result. It is clear that the result of QGSP_BERT is lower than other results in both neutron and photon flux. This is especially clear in the high- $R$ region $(R=1000 \mathrm{~cm})$ where the neutron and photon fluxes are 5 and 20 times lower, respectively, than the result of FLUKA-FLUGG. The other two results, FLUKA-FLUGG and QGSP_BERT_HP, are similar for both fluxes. The QGSP_BERT_HP/FLUKA-FLUGG flux ratios are compared and mapped in Figure 3. For both photon and neutron fluxes, the ratios are close to 1 in most regions of the ATLAS cavern. Of the 28,800 mesh points in the neutron flux, there are 5 points where the ratio is larger than 10 . These points are sparsely populated, with fewer than 5 jobs per 1,000 having non-zero entries. Therefore we can expect large statistical errors. However FLUKA-FLUGG results are higher in all cases, and all points are located in the well-shielded area. Differences between the physics of Geant4 and FLUKA cannot be excluded from this calculation. All photon flux ratios are within a factor of 5 . We also examined the flux of photons $>1 \mathrm{MeV}$, but no difference was observed in the comparison map of all photon energies.

Figure 4 is a histogram of the ratios. The horizontal axis is logarithmic so that 0.5 and 2 are equidistant from 1 . The labels $+/-100 \%$ and $41 \%$ indicate a ratio range of $1 / 2$ to 2 and $1 / \sqrt{2}$ to $\sqrt{2}$, respectively. The distributions are centered on 1 in all cases. Table 1 shows the percentage of mesh points that have ratios within these ranges. More than $90 \%$ of mesh points have ratios within $41 \%$, and 98 to $99 \%$ of mesh points fit 


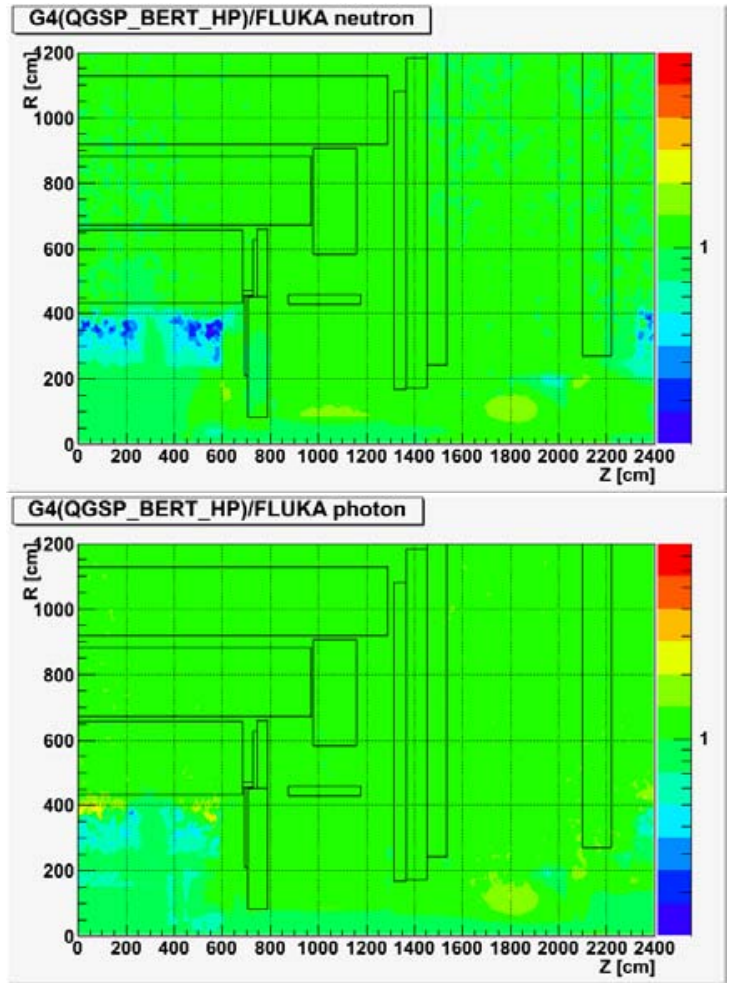

Figure 3. Ratio map of neutron (upper panel) and photon (lower panel) flux of Geant4-QGSP_BERT_HP to FLUGG-FLUKA calculations. The upper and lower limit of color scale is 8 and 0.125 resnectivelv.

within the $100 \%$ (difference of factor 2). Considering the scale in size and weight, the variety of materials and the complexity of the ATLAS geometry, the agreement between the two results is remarkable.

\section{Conclusion}

A FLUGG application for ATLAS cavern background simulation was developed. A new geometry for the application was built from scratch. The geometry and FLUGG technology presents a unique opportunity for unbiased comparison between FLUKA and Geant4 for reasonably realistic, large, modern detector used in high energy physics experiments. Excepting for area close to the beam pipe the G4 result from the QGSP_BERT physics gives much lower flux than the other results. The result of QGSP_BERT_HP, which uses the High Precision neutron model for low energy neutron transportation, is similar to the FLUKA result. Neutron and photon fluxes were compared throughout the entire ATLAS cavern and the differences between FLUKA and Geant4 are within +/- $41 \%$ in most regions.

\section{References}

[1] ATLAS collaboration, G. Aad et al. The ATLAS Experiment at the CERN Large Hadron Collider, JINST 3 S08003 2008

[2] S. Baranov, M. Bosman, I. Dawson, V. Hedberg, A.Nisati and M.Shupe, Estimation of radiation

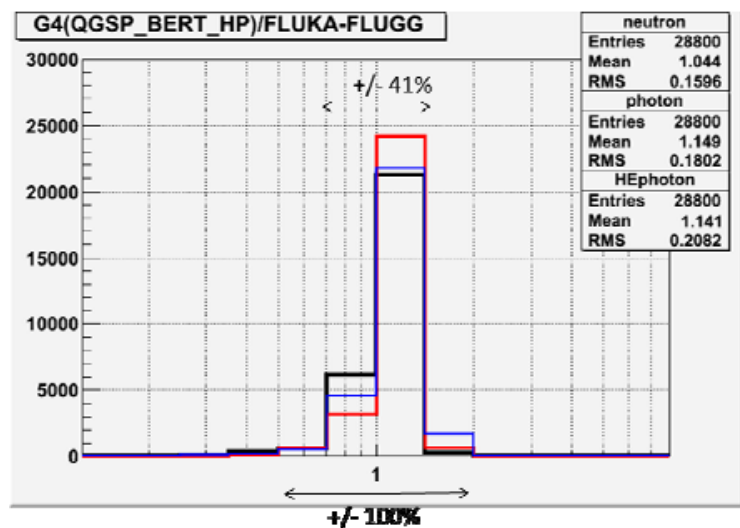

Figure 4. Distribution of the ratios of neutron (black), all energy photon (red) and photon $>1 \mathrm{MeV}$ (blue) flux of Geant4 QGSP BERT HP to FLUKA-FLUGG

Table 1. The ratio of the meshes that the difference of the flux between FLUKA-FLUGG and Geant4 QGSP_BERT_HP is within ranges

\begin{tabular}{ccc}
\hline Quantity & Within 41\% & $\begin{array}{c}\text { Within100\% } \\
(\%)\end{array}$ \\
\hline Neutron flux & 95 & 98 \\
Photon flux & 95 & 99 \\
Photon>1MeV flux & 91 & 99 \\
\hline
\end{tabular}

background, impact on detectors, activation and shielding optimization in ATLAS, Atlas Radiation Background Task Force Summary Document, ATL-GEN-2005-001 2005.

[3] R. Engel, Photoproduction within the two component Dual Parton Model: Amplitudes and cross sections, Z. Phys. C 66 (1995), pp. 203-214.

[4] H. Hirayama, Intercomparison of medium-energy neutron attenuation in iron and concrete (8), Nuclear Science Shielding Aspects of Accelerators, Targets and Irradiation Facilities - SATIF 10 Workshop Proceedings, Geneva, Switzerland 2-4 June 2010, Nuclear Energy Agency - Nuclear Science, OECD Publishing 2010, pp. 217-233, ISBN 9789264034679.

[5] G. Battistoni, S. Muraro, P.R. Sala, F. Cerutti, A. Ferrari, S. Roesler, A. Fasso` and J. Ranft, The FLUKA code: Description and benchmarking, Proceedings of the Hadronic Shower Simulation Workshop 2006, Fermilab 6-8 September 2006, M. Albrow, R. Raja eds., AIP Conference Proceeding 896, (2007), pp. 31-49; A. Ferrari, P.R. Sala, A. Fasso' and J. Ranft, FLUKA: A Multi-particle Transport Code, CERN-2005-10 (2005), INFN/TC_05/11, SLAC-R-773.

[6] S. Agostinelli et al., Geant4-a simulation toolkit, Nuclear Instruments and Methods in Physics Research Section A: Accelerators, Spectrometers, Detectors and Associated Equipment, 506 (2003), pp. 250-303; J. Allison et al., Geant4 developments and applications, Nuclear Science, IEEE Transactions 53 (2006), pp. 270- 278. 